\title{
Hipertensão Arterial Sistêmica: a Perspectiva dos Docentes no Ensino Médico
}

\section{Systemic Arterial Hypertension: the View of Teachers in Medical Training}

\author{
Alessandro da Silva Scholze $e^{1 \odot}$ \\ Lucas Zago Scopel \\ Priscila Soranzo Zappelini ${ }^{1}$ \\ Carlos Francisco Duarte Júnior
}

\section{PALAVRAS-CHAVE}

- Hipertensão.

- Docentes.

- Educação Médica.

- Percepção Social.

- Conhecimentos, Atitudes e Práticas em Saúde.

\begin{abstract}
RESUMO
Investigamos as representações sociais dos professores do curso de Medicina de uma universidade no litoral norte de Santa Catarina quanto à abordagem de pessoas com hipertensão arterial sistêmica (HAS) na prática clínica. Realizamos uma pesquisa qualitativa, utilizando entrevistas semiestruturadas para a coleta de dados com oito docentes do curso em uma amostragem de conveniência, representando as grandes áreas envolvidas no internato médico. Os dados foram analisados conforme a metodologia do Discurso do Sujeito Coletivo, usando como operadores as idéias centrais e expressões-chave. Verificamos que as pessoas com hipertensão chegam aos profissionais de diversas formas: a partir de um achado no exame físico realizado por outros motivos, na revisão de saúde por questões preventivas, em situações de urgência, referências ou com queixas que a pessoa atendida relaciona à pressão arterial elevada. Os docentes identificaram falta de associação entre a HAS e sintomas, atribuindo tal associação às pessoas acometidas, ainda que o discurso dos próprios sujeitos tratasse a HAS como doença mais do que como fator de risco. A má adesão ao tratamento surgiu como limitação das medidas não farmacológicas e farmacológicas, evidenciando-se a dificuldade de justificar as mudanças exigidas na vida das pessoas para lidar com um risco que não se traduz em illness na ausência de complicações associadas. Sugere-se a importância de definir de forma adequada a HAS como um sinal vital e fator de risco na graduação médica, a fim de evitar a promoção da doença e abordar de forma efetiva o problema por meio de um Plano de Manejo Conjunto quando as intervenções se fizerem necessárias.
\end{abstract}




\section{KEY-WORDS}

- Hypertension.

- Faculty.

- Medical Education.

- Social Perception.

Recebido em: 23/2/19

Aceito em: 7/4/19

\begin{abstract}
We investigated the social representations of medicine teachers from a University in Santa Catarina state regarding their clinical approach to people with hypertension. This was a qualitative study employing semi-structured interviews with a convenience sample of eight teachers, each representing a field included in undergraduate medical internship. The collected data were analyzed according to the Collective Subject Discourse (DSC) methodology applying the resources of central ideas and key expressions. We verified that people with hypertension are identified by the subjects when they seek medical attention for different purposes: medical examination for complaints not related to hypertension, health check-ups, emergency situations, referrals from other professionals or even complaints that the person attributes to hypertension. The medical teachers identified a lack of association between hypertension and symptoms, attributing that association to those afflicted with hypertension, even though the subjects talked of hypertension more like as a disease, than a risk factor. Poor treatment adherence emerged as a limitation of non-pharmacological measures as much as of the pharmacological ones, pointing to the difficulties involved in justifying the lifestyle modifications usually prescribed to deal with a risk factor that is not identified as an illness when there are no associated complications defining disease situations. We suggest the importance of adequately defining hypertension as a risk factor throughout medical undergraduate training, in order to avoid disease mongering. Hypertension must be approached applying a joint problem management plan involving the physician and the person with hypertension.
\end{abstract}

\section{INTRODUÇÃO}

A formação médica é objeto de constante discussão, refletindo controvérsias mais amplas que envolvem a visão que a sociedade constrói da saúde. Para esse tema convergem questões éticas, mudanças constantes no conhecimento científico, além dos interesses econômicos e políticos de diferentes grupos sociais ${ }^{1}$.

Um exemplo no qual podemos observar tais aspectos da construção do conhecimento científico na medicina é a forma como a hipertensão arterial sistêmica (HAS) é descrita, compreendida e ensinada no ambiente acadêmico e na prática médica. Esse exemplo é pertinente devido a sua magnitude como problema de saúde, já que, no contexto de doenças crônicas, a HAS se apresenta em mais de $30 \%$ da população adulta e é fator de risco para importantes comorbidades, como falência renal, acidente vascular cerebral (AVC) e infarto agudo do miocárdio (IAM) ${ }^{2,3}$.

A HAS é um fator de risco assintomático, definida como níveis iguais ou superiores a $140 \mathrm{mmHg}$ de pressão sistólica e/ ou iguais ou superiores a $90 \mathrm{mmHg}$ de diastólica, excetuando-se os casos em que cursa com crises hipertensivas envolvendo lesões de órgãos-alvo em emergências ${ }^{2,3}$.

Já para as pessoas com HAS, mais marcantes que as definições quantitativas são as Representações Sociais (RS) de saúde e doença vivenciadas no cotidiano, que trazem consigo uma interação do individual com o social. As RS de saúde e doença apresentam-se como fortes concepções e, por vezes, são tomadas como realidade, o que traz consequências como a percepção dos profissionais da saúde sobre hipertensão no sentido de que essa condição marca o fim de uma vida normal em face das mudanças de estilo de vida que exige. Especialmente, a representação dos profissionais de saúde com relação à HAS, mesmo na atenção básica, conduz a um conceito de doença, em lugar de fator de risco. Os profissionais descrevem que a HAS tem controle como uma doença crônica, permitindo uma vida normal no contexto familiar e do trabalho, mas as orientações médicas não são seguidas de modo a obtê-lo. Apesar disso, poucos estudos investigaram as Representações Sociais dos profissionais da saúde acerca das pessoas com HAS e de sua terapêutica, englobando fatores a ela relacionados, como adesão e eficácia ${ }^{4,5}$.

Por sua vez, as concepções dos enfermos acerca de sua situação, a partir de suas RS, são essenciais na adesão ao tratamento, principalmente porque a HAS é uma condição crônica, cujo tratamento envolve mudanças no estilo de vida e na dieta, elementos enraizados na cultura, mas é vista como uma condição de "não doença" pelas pessoas que vivem com hipertensão e se consideram saudáveis ${ }^{6}$. Ainda, a hipertensão 
pode ser vista como uma forma de traduzir o sofrimento psicossocial vivenciado por diferentes motivos no contexto social, profissional ou familiar. Essa percepção de que a HAS é um problema sintomático decorrente daquilo que é descrito popularmente como "nervoso" conduz ao entendimento de ser também um problema limitado no tempo e potencialmente curável, ainda mais quando, mesmo sem tratamento, eventualmente são verificados níveis pressóricos normais em pessoas diagnosticadas como tendo $\mathrm{HAS}^{7}$.

Como visto, as Representações Sociais dos profissionais de saúde têm implicações práticas, uma vez que interferem, direta ou indiretamente, no modo de atuação quanto ao manejo clínico dos portadores de HAS. Igualmente, ao atuarem no ensino médico, esses mesmos profissionais podem propagar suas RS, reproduzindo através dos discentes uma atuação conforme a prática decorrente de tais representações.

Assim, o presente artigo procurou analisar as Representações Sociais em relação à HAS na prática clínica dos docentes do curso de Medicina de uma universidade do litoral norte de Santa Catarina. Para isso, identificamos, na perspectiva dos docentes, o que costuma fazer com que as pessoas com HAS procurem a atenção médica, que sintomas esses profissionais atribuem às pessoas com HAS e, por fim, como as características das pessoas hipertensas poderiam influenciar na adesão ao tratamento, na visão dos professores de Medicina.

\section{CONSIDERAÇÕES METODOLÓGICAS E ÉTICAS}

Este estudo, de cunho qualitativo e caráter exploratório, contou com a participação de oito docentes médicos do curso de Medicina de uma universidade do litoral norte de Santa Catarina, cujas disciplinas envolvessem temas relacionados com a HAS em atividades teóricas ou práticas, atuando no internato médico durante o segundo semestre de 2015. Cada docente representou uma das seguintes áreas: Medicina de Família e Comunidade (MFC), Nefrologia, Geriatria, Clínica Médica, Cirurgia Geral, Ginecologia e Obstetrícia, Medicina Intensiva e Pediatria. As áreas Básica e de Saúde Mental não foram incluídas na amostragem, uma vez que não tinham representação específica no internato médico desse curso, sem prejuízo da representatividade da amostra, uma vez que as áreas definidas a partir do internato compreendiam os docentes que habitualmente lidam com a prática clínica envolvendo situações de HAS. Essa amostra de conveniência definiu, assim, um grupo de informantes-chave que foram identificados com base nas informações fornecidas pela coordenação do curso nos planos de ensino das respectivas disciplinas.

Uma vez que os entrevistados concordaram com a participação na pesquisa e assinaram o Termo de Consentimento
Livre e Esclarecido (TCLE), agendamos a entrevista de acordo com a disponibilidade dos entrevistados. Foi dada preferência à condução da entrevista no local de trabalho do professor, uma vez que consideramos esse contexto como o mais representativo da sua abordagem das pessoas com hipertensão. Conduzimos entrevistas semiestruturadas, que foram gravadas digitalmente para posterior transcrição e análise, contando com a presença de dois dos pesquisadores, a fim de garantir a fidedignidade das transcrições, que foram revisadas por ambos.

Os dados transcritos das entrevistas foram analisados conforme a metodologia do Discurso do Sujeito Coletivo (DSC), usando-se dois operadores: as ideias centrais e as expressões-chave. Na construção do DSC, buscamos, de início, as representações que os informantes apresentaram individualmente a partir de cada pergunta do roteiro de entrevista, as quais foram elaboradas com base nos objetivos específicos do estudo. Para isso, conduzimos uma primeira leitura de cada entrevista a fim de apreender seu conteúdo de forma mais geral, seguindo-se uma leitura mais aprofundada, buscando as ideias centrais expressas pelo entrevistado individualmente, que descrevem suas representações a respeito do tema. Tais ideias centrais são representadas na sua fala por palavras ou trechos significativos que servem de prova empírica: as expressões-chave, constituídas por trechos selecionados dos depoimentos que sedimentam as ideias centrais ${ }^{8}$.

Nesse momento, foi construído o Instrumento de Análise do Discurso 1 (IAD1), tendo sido obtido um IAD1 para cada entrevistado, listando-se as diversas ideias centrais presentes na sua fala e as respectivas expressões-chave. Uma vez concluído esse passo para todos os entrevistados, as ideias centrais identificadas para cada entrevistado foram revisadas e, sendo equivalentes entre os diferentes sujeitos, reunidas, com as expressões-chave correspondentes, em um Instrumento de Análise do Discurso 2 (IAD2). Neste, todas as expressões-chave presentes nas falas dos diferentes entrevistados foram listadas sob a ideia central que representavam, a qual pode ter sido comum a mais de um sujeito. Por fim, as expressões-chave foram apresentadas em um Discurso do Sujeito Coletivo, de modo organizado e sem redundâncias, a fim de ilustrar as ideias centrais evidenciadas pelos entrevistados em relação a cada uma das questões elaboradas originalmente no roteiro da entrevista ${ }^{8}$.

Sintetizadas as falas de todos os indivíduos entrevistados, reunindo diferentes fragmentos dos discursos individuais, estes foram transformados em uma macroproposição. Desse modo, o DSC constituiu-se daquilo que um entrevistado respondeu e das contribuições feitas por outros membros da mes- 
ma coletividade, mas que poderiam ter sido daquele primeiro, já que todos compartilhavam as mesmas representações.

Este trabalho foi avaliado e aprovado por Comitê de Ética em Pesquisa, com o parecer de número 1.177.930, sendo conduzido conforme as recomendações da resolução 466/2012 do Conselho Nacional de Saúde.

\section{RESULTADOS}

As oito entrevistas semiestruturadas foram transcritas e arranjadas em três DSC, cada qual correspondente a um dos três questionamentos que nortearam as entrevistas (Quadros 1 a 3). As idades dos oito médicos entrevistados variaram entre 34 e 53 anos, sendo seis participantes do sexo masculino e dois do feminino. O tempo de atuação na profissão médica de cada sujeito era de 6 a 28 anos, e o tempo de docência, de 2 a 17 anos. Todos os profissionais entrevistados responderam aos três questionamentos norteadores.

QUADRo 1
DSC 1 - Motivo(s) que leva(m) os portadores
de HAS a procurar a atenção médica, na visão
dos docentes do curso de Medicina

\section{QUADro 2}

DSC 2 - Sintoma(s) atribuído(s) pelos docentes

\section{do curso de Medicina aos portadores de HAS}

A hipertensão é uma doença silenciosa. Noventa por cento dos pacientes não vão ter sintoma algum. A cefaleia é uma das coisas que levam o paciente ao atendimento pensando que tem pressão alta, e sua relação causa-efeito é uma coisa muito duvidosa. Inclusive, a literatura diz que não tem relação muito clara, por isso peço aos pacientes que não façam essa associação. Os pacientes que eu venho acompanhando há muito tempo se dão conta: eles estão com dor de cabeça, vão medir a pressão e está normal; não estão sentindo nada, vão medir e a pressão está alta.

\section{QUADro 3}

DSC 3 - Característica(s) dos portadores de HAS

que influencia(m) na adesão ao tratamento, na visão dos docentes do curso de Medicina

O fato de a hipertensão ser uma doença assintomática atrapalha. As complicações são muito tardias, e o paciente acredita que eventos cardiovasculares não vão ocorrer. O nível cultural do paciente interfere com a adesão. Se inventassem uma medicação anti-hipertensiva que fosse para ser tomada de seis em seis meses, teria grande adesão. À medida que você aumenta o número de medicações, a aderência é pior. Outra coisa que interfere na adesão são os efeitos adversos e as necessárias mudanças de estilo de vida. As mudanças de estilo de vida são difíceis, principalmente a prática de atividade física, retirar o sal da dieta e a questão do uso de álcool. A gente já vai meio derrotado, porque só uma minoria realmente as faz. No sexo masculino a aderência é pior do que nas mulheres, em parte pelo maior consumo alcoólico. Para uma boa adesão, é preciso entender o paciente como um todo e fazer com que ele tenha acesso à consulta médica. É preciso ter um diálogo que o faça entender o que é HAS, seus malefícios e os benefícios que ele vai ter por não ter complicações agudas ou crônicas. Acho que a atenção primária é o espaço para tratar hipertensão. Nas gestantes a adesão é de 100\%. Elas têm vontade de fazer o tratamento, pois querem cuidar do neném e têm muito medo de convulsionar.

\section{DISCUSSÃO}

A busca pela verificação da pressão arterial (PA) pode fazer parte de itinerários de cuidado pessoal, como citado no DSC 1 (Quadro 1): "para uma revisão em saúde, um check-up" e "porque medir a pressão é um hábito local e uma forma de cuidar de si". Funciona como uma atitude de prevenção e preservação da saúde não apenas no âmbito individual, mas também como parte de alguma forma de rastreamento, geralmente não programático ${ }^{2}$ : "a detecção ao acaso dá-se em campanhas, em exames médicos do trabalho, em exames clínicos pré-operatórios ou no pré-natal" (Quadro 1). Nota-se que a RS de que a aferição da PA faz parte dos cuidados com a saúde não se limita aos acometidos, mas abrange também os profissionais de saúde e elaboradores de campanhas de medidas ou rastreamentos. Nesse sentido, o DSC 1 não parece fazer diferença entre situações de rastreamento ou cuidados continuados com situações crônicas relacionadas à HAS e a verificação da PA como sinal vital de forma rotineira nas pré-consultas ou consultas por outros motivos.

O DSC 1, no que diz respeito à percepção da HAS pelos docentes de Medicina em sua apresentação inicial, reúne uma série de condições nas quais a HAS é identificada: como fator de risco, em urgências/emergências hipertensivas ou em quadros de lesões de órgão-alvo cronicamente estabelecidas. Assim, tais condições diversas podem acabar confundidas entre si, como ao se apontar que as crises hipertensivas "podem ser desencadeadas por grande consumo de alimentos salgados, bebidas alcoólicas ou grande estresse pessoal" (Quadro 
1). Na literatura, as crises hipertensivas correspondem a um grupo heterogêneo de situações sintomáticas, caracterizadas por lesão aguda de órgão-alvo, juntamente com elevação da PA, muitas vezes confundidas com pseudocrises hipertensivas, estas corretamente consideradas resultantes do estresse pessoal e que também devem ser diferenciadas da elevação da PA em pessoas com HAS, assintomáticas, as quais não seguem o tratamento, mantendo, por exemplo, o consumo elevado de sal e álcool. A HAS tem um papel de fator de risco para a lesão de órgão-alvo, não sendo causa das crises hipertensivas, tanto que nessas situações sua redução deve ser cuidadosa para evitar a piora das lesões isquêmicas habitualmente encontradas nas crises ${ }^{9}$.

Há significantes diferenças no modo de percepção de uma condição clínica pela pessoa afetada e pelo profissional que a trata. A antropologia médica vem utilizando os termos illness e disease, respectivamente, para descrever o modo como o acometido experimenta a condição de doença e o modo como o profissional de saúde vê o adoecimento. Illness diz respeito à experiência pessoal e subjetiva da doença, trazendo consigo os sentimentos a respeito da situação vivida, as ideias criadas a respeito do infortúnio sofrido, a percepção das mudanças na funcionalidade da pessoa em seu cotidiano e as expectativas quanto a soluções para o problema. Incluem-se aqui as influências do contexto, as experiências prévias pessoais com problemas de saúde ou de pessoas próximas, que também contribuem para a interpretação que o sujeito elabora sobre sua experiência com a doença. Por sua vez, o conceito de disease aponta as definições biomédicas da doença, empregadas pelos profissionais da saúde. Ainda que úteis, estas definições são necessariamente reducionistas e generalizantes, modelos teóricos no mais das vezes baseados na mensuração de variáveis quantitativas que delimitam o que é normal ou não nas estruturas ou funções do corpo. Ainda que ambos os conceitos estejam intrinsecamente relacionados, a competência do profissional de saúde para identificar tais divergências nas formas de compreender, descrever e lidar com as situações de doença pode contribuir para uma melhor comunicação com a pessoa atendida. Isso é ainda mais evidente em situações nas quais disease e illness não são concomitantes, como se dá com a HAS e outros fatores de risco ${ }^{6,10}$.

Como exposto no DSC 3 (Quadro 3), o entendimento pelos profissionais de saúde a respeito da HAS como uma condição que deve ser tratada, quando confrontada com a não execução ou manutenção desse tratamento pelos portadores, na maioria das vezes assintomáticos, expõe uma divergência entre ambas as partes. A RS dos acometidos de que a PA elevada não é uma doença ou apenas o é quando relacionada a algum evento agudo pode determinar uma má adesão ao tratamento crônico, uma vez que não há a visão positiva de custo-benefício por aquele que terá de realizar mudanças de estilo de vida como parte do tratamento. Isto se confirma quando, ao se verificar uma situação de doença hipertensiva específica da gestação no pré-natal, garante-se adesão massiva das mulheres ao tratamento proposto: “Nas gestantes, posso dizer que a adesão é de 100\%" (Quadro 3) ${ }^{6,10}$.

Tal feito pode explicar por que "as mudanças de estilo de vida são difíceis" e por que "só uma minoria realmente as faz" (Quadro 3). Os acometidos pela hipertensão veriam mais malefícios na execução das mudanças de estilo de vida e de hábitos enraizados cultural e socialmente, conforme recomendadas pelos médicos, do que na condição de hipertensão propriamente dita, por não se sentirem doentes e terem concepções de adoecimento e saúde diferentes das dos médicos ${ }^{6,11}$. Em um estudo sobre as representações sociais de profissionais da Estratégia Saúde da Família a respeito da HAS, a identificação das dificuldades para mudanças no estilo de vida apontava tanto os conflitos que as tentativas de mudança geram nas famílias, diante da convivência com pessoas sem hipertensão e que não precisavam realizar tais mudanças, gerando falta de apoio, bem como uma "culpabilização das vítimas", ao considerar os portadores de HAS individualmente responsáveis pela adesão ou não ao tratamento, em especial no que concerne ao abandono de vícios e modificação de outros fatores de risco associados, como sedentarismo ou obesidade ${ }^{5}$.

O DSC dos docentes afirma que "o fato de a hipertensão ser uma doença assintomática atrapalha" e "é preciso ter um AVC ou um infarto para ele voltar com a medicação" (Quadro 3). E, mesmo que o DSC 2 (Quadro 2) aponte ser a HAS "uma doença silenciosa, sem causas de dor, assintomática", sugere-se que alguns portadores atribuem à hipertensão sintomas como cefaleia, tontura, dor na nuca e mal-estar, sendo estes os motivos da consulta médica (Quadro 1). Também no discurso dos docentes, a definição da HAS como doença e, portanto, passível de se apresentar com sintomas, evidencia-se na dúvida exposta no DSC 2: "[...] inclusive, na literatura, diz que não tem relação (entre HAS e sintomas) muito clara". Na verdade, a questão de a HAS ser ou não sintomática, na relação entre HAS e cefaleia, por exemplo, já foi investigada e descartada em estudos clássicos, no que se refere à HAS não complicada por lesões de órgão-alvo, como o U. S. Health Examination Survey of Adults (Nhanes), conduzido a partir de 1960-1962 ${ }^{12}$. Da mesma forma, a alegada capacidade de alguns hipertensos predizerem um aumento na pressão sanguínea de forma espontânea pela simples interpretação de sinais e sintomas foi avaliada em 1997 por Cantillon et al. ${ }^{13}$, que não encontrando 
resultados que a comprovassem. Mais recentemente, em 2008, Tronvik et al. ${ }^{14}$ também não encontraram relação da HAS com sintomas, sugerindo inclusive que pessoas com níveis elevados de pressão arterial apresentavam $40 \%$ menos probabilidade de terem dores de cabeça em comparação com aqueles de níveis normais. Por fim, um estudo transversal com 33.105 indivíduos propôs que a mera consciência da pessoa acometida em ser portadora da condição de HAS, por si só, independentemente de outros fatores, contribui para elevados níveis de patologias emocionais, com sintomas de depressão e ansiedade, potencialmente confundidos com manifestações da própria $\mathrm{HAS}^{15}$.

Tal persistência na busca por associações entre a HAS e sintomas não apenas por parte das pessoas com HAS mas especialmente pelos médicos, como mostra a recorrência do tema na literatura médica desde a segunda metade do século XX, parece apontar uma Representação Social em que a HAS é vista como doença e não como fator de risco. Evidencia-se, ainda, que a ausência de validação empírica dessa RS não foi suficiente para modificá-la na prática, ignorando-se as evidências científicas acumuladas. Neste ponto, as RS dos docentes e aquelas identificadas entre os portadores de HAS coincidem quanto à expectativa de que a hipertensão seja uma condição sintomática, ainda que entre os portadores isto pareça ser compreendido como uma manifestação somática do "nervoso" criado pelas condições adversas do contexto, enquanto entre os médicos pode ser visto como uma tentativa reducionista de enquadrar a complexidade do sofrimento de um indivíduo a partir de uma medida quantitativa específica, a qual o profissional considera passível de tratamento dentro da sua competência ${ }^{7,16}$.

Neste caso, a HAS é percebida como doença diante da limitação da medicina para lidar com a condição de incerteza inerente aos fatores de risco, como é o caso da HAS, e da pressão social para medicalizar os eventos da vida, incluindo aí a variável fisiológica da pressão arterial, sujeita a modificações cotidianas diante de situações psicossociais adversas ${ }^{2,16}$.

O poder do exercício médico em classificar condições clínicas atua estabelecendo o limiar entre doença e "não doença", entre enfermidade, saúde e fator de risco. Por vezes, porém, erroneamente atribui a condições passíveis de ponderação, quanto à necessidade de intervenção, uma estrita e imperativa atuação diagnóstica ou terapêutica. Pelo modo como entendem a HAS, como doença ou fator de risco, podem atribuir à investida terapêutica maior ou menor ênfase. É assim que atividades médicas se tornam potencialmente danosas ao se dirigirem a fatores de risco como se fossem enfermidades bem definidas, com evolução patológica totalmente conheci- da e inevitável caso não seja instituído o tratamento ${ }^{16}$. Pelo contrário, uma vez que o tratamento se dirige a um fator de risco, torna-se evidente a necessidade de garantir benefícios potencialmente maiores que os possíveis danos de quaisquer intervenções, especialmente nos níveis de prevenção primária e secundária, como é o caso do manejo habitual da HAS ${ }^{17}$. Assim, em "é preciso ter um diálogo que o faça entender o que é HAS, os malefícios e os benefícios que ele vai ter por não ter complicações" (Quadro 3), supõe-se, de forma enganosa, que o tratamento do fator de risco elimina a possibilidade do desfecho indesejado.

A prevenção quaternária, como proposta por Jamoulle e Gomes ${ }^{17}$, atua nesse sentido, pois "outra coisa que interfere na adesão são os efeitos adversos" (Quadro 3), como sonolência e disfunção erétil, sem que haja benefício garantido do uso das medicações, uma vez que a HAS não é uma doença, mas um fator de risco correlacionado estatisticamente com o aumento na ocorrência de eventos cardiovasculares, sem fazê-lo de modo determinístico e inexorável ${ }^{2,3}$. A transformação arbitrária de um fator de risco em doença quebra o dever implícito com o paciente e com a sociedade, de precaução extrema nas intervenções de níveis de prevenção primária e secundária, por violar o princípio fundamental de benefícios superando os riscos: primo non nocere. O faz ao elevar a categoria "benefício" a um patamar artificialmente superior, sugerindo que a instituição da intervenção deve evitar com certeza um desfecho desfavorável, o que é impossível de se afirmar no nível individual, em que o risco é pequeno; e mesmo pessoas nas quais se identificam múltiplos fatores de risco podem nunca vir a sofrer o evento a ser evitado pelo tratamento dos fatores de risco, morrendo de outras causas, ainda que não se submetam à intervenção proposta. Por outro lado, qualquer intervenção medicamentosa traz consigo, inevitavelmente, um acréscimo de risco para a pessoa, na forma de eventos adversos. Logo, a Representação Social de que a HAS é uma doença que deve, inquestionavelmente, ser alvo de intervenção médico-terapêutica tem grande efeito de promoção da doença, podendo resultar em iatrogenia ${ }^{16,17}$.

A exemplo do reconhecimento da hipertensão arterial como fator de risco, surgem questionamentos quanto ao real benefício terapêutico farmacológico para HAS ditas "leves" ou mild hypertension, cujos níveis pressóricos não extrapolam $159 \mathrm{mmHg}$ de pressão sistólica e $99 \mathrm{mmHg}$ de pressão diastólica, em pessoas sem comorbidades ou outros fatores de risco. Sugere-se que os "hipertensos leves" não deveriam receber medicações anti-hipertensivas na ausência de doenças de órgãos-alvo estabelecidas ou alto risco cardiovascular. Mesmo assim, grande parte dos "hipertensos leves" está, de fato, re- 
cebendo tratamento medicamentoso para controle da pressão arterial $^{18}$.

Para além do cenário clínico-prático, a concepção de que a HAS é uma doença e que sua permanência sem tratamento resultará, deveras, em um evento cardiovascular possui consequências para o ensino médico, sobretudo na graduação. Uma vez que as RS dos professores tomam a HAS por doença, em lugar de fator de risco, sua participação na formação dos alunos de graduação potencialmente perpetuará tal Representação, de acordo com a qual a HAS é considerada uma doença e, como tal, sempre é justificadamente tratada com medicamentos e revertida a todo custo. Essas RS podem propagar um equívoco, com o potencial de perpetuar a promoção da doença em pessoas abordadas pelos receptores da Representação que estabelece erroneamente a HAS como doença ${ }^{2,3}$.

O DSC 3 expõe, ainda, características relativas à HAS e aos portadores que implicariam menor aderência terapêutica. A baixa adesão é identificada como a principal causa do controle inadequado da pressão arterial. Pelo fato de a hipertensão ser crônica, "algo a longo prazo" (Quadro 3), o abandono do tratamento e a baixa adesão seriam ainda maiores, assim como nos casos em que há maior número de medicações a utilizar e/ou maior frequência nas tomadas diárias das medicações ${ }^{19}$. Em estudos sobre o tema, não se encontram diferenças de adesão por variações de gênero, em confronto com a afirmação de que "(os pacientes) do sexo masculino têm aderência pior do que as mulheres" (Quadro 3). Na realidade, as mulheres talvez apresentem mais baixa adesão que os homens por estarem insatisfeitas com a comunicação com seu médico e por experimentarem sintomas depressivos com o uso da medicação $0^{20}$. Aqueles com baixa escolaridade, como referido no DSC 3 , tendem a ser menos aderentes, e a relação entre o portador de hipertensão e a equipe de saúde influencia na adesão. Especificamente quanto ao tratamento farmacológico, ressaltam-se os problemas de custo, efeitos indesejáveis e esquemas terapêuticos complexos, como também exposto no DSC 3 em "à medida que você aumenta o número de medicações, a aderência é pior" e "se inventassem uma medicação que fosse para ser tomada de seis em seis meses, teria grande adesão"2,11.

Diante das dificuldades na adesão percebidas pelos docentes no tratamento de um fator de risco como a HAS, observa-se a utilidade de empregar o Método Clínico Centrado na Pessoa ${ }^{10}$, como se depreende do DSC 3: (1) avalie-se tanto a doença/fator de risco quanto a experiência de seu portador (illness) antes de traçar uma meta terapêutica; (2) entenda-se o contexto em que a pessoa está inserida e suas concepções e preferências individuais, de modo a adequar as propostas de tratamento àquela pessoa em particular, melhorando a ade- são por meio de diálogo e entendimento mútuo - "é preciso entender o paciente como um todo"; (3) elabore-se um plano conjunto, de acordo com o componente anterior, por meio da discussão, exposição realista de fatos e da participação do paciente hipertenso em sua decisão terapêutica para "ter um diálogo que o faça entender o que é HAS"; (4) intensifique-se a relação médico-paciente, havendo um comprometimento recíproco entre a instituição do tratamento e o seu cumprimento, firmado em uma troca de confiança e com sensação de propósito; por isso, "a atenção primária é o espaço para tratar hipertensão, desde que o profissional tenha tempo e esteja disponível". Nesse sentido, o entendimento da experiência da doença do acometido (illness) pelo profissional de saúde pode contribuir para um cuidado baseado na integralidade, mais humanizado e, espera-se, mais efetivo.

É relevante, por sua ausência no DSC dos docentes, a implicação de fatores relacionados ao atendimento e à acessibilidade na má adesão, apenas apontando-se no DSC 3 que "para uma boa adesão, é preciso entender o paciente como um todo e fazer com que ele tenha acesso à consulta médica". Usuários do Sistema Único de Saúde (SUS) podem encontrar dificuldades no acesso a unidades de saúde, por motivos geográficos, burocráticos ou de funcionamento da própria unidade ${ }^{21}$. Desse modo, pessoas hipertensas que vivem em regiões afastadas dos serviços de saúde, bem como aquelas que não tomam conhecimento da existência de unidades de saúde próximas às suas residências podem acabar por não terem respeitados seus direitos de acesso com equidade aos cuidados para HAS. Igualmente, a prescrição, pelo médico assistente, de medicamentos não disponíveis no programa de distribuição gratuita mantido pelo governo, apesar da ampla variedade de anti-hipertensivos englobados, pode resultar em mais um motivo para não aderência ao esquema medicamentoso proposto, acordando com a constatação de que o nível socioeconômico é fator de baixa adesão².

Considera-se também importante ressaltar o fato de a HAS iatrogênica não ter sido citada, principalmente quando se aponta que o cuidado com a mulher em diversas ocasiões se faz pelo médico ginecologista, muitas vezes o único profissional de saúde com quem ela terá contato: "quando o ginecologista é o único médico que algumas mulheres procuram, ele acaba fazendo o controle pressórico" (Quadro 1). Nessa situação, os anticoncepcionais orais combinados com estrógenos são listados entre as medicações a serem verificadas como potenciais causadoras da elevação da pressão arterial $^{2}$. No que se refere ao uso de estrógenos no planejamento familiar e outras condições, com suas potenciais repercussões na saúde da mulher, ressalta-se a necessidade de implementar um cuidado 
clínico centrado na pessoa, visando compreendê-la de forma abrangente, idealmente a partir da Atenção Primária em Saúde, uma vez que no Brasil a HAS é a contraindicação mais comum para o uso de estrógenos, presente em 15,1\% das usuárias de anticoncepcionais orais ${ }^{22}$.

\section{CONSIDERAÇÕES FINAIS}

Conhecer as RS dos docentes de Medicina em relação à hipertensão na sua prática clínica constitui um elemento fundamental para compreendermos as implicações para as pessoas identificadas com (e talvez mesmo para aquelas sem) HAS e o sistema de saúde. Esse conhecimento também é essencial para o ensino médico, que tenderá a reproduzir tais RS e suas consequências para a sociedade, uma vez que, como docentes, os sujeitos deste estudo representam o modelo para os profissionais hoje em formação no curso de Medicina.

As pesquisas voltadas a compreender as RS dos profissionais de saúde, especialmente dos médicos, a respeito da HAS são escassas, o que talvez reflita uma pré-concepção segundo a qual a perspectiva profissional se fundamenta exclusivamente nas evidências científicas sobre o tema, alheia a outras influências do contexto social. No entanto, como qualquer construto social, a abordagem médica da HAS é produto de um amplo conjunto de conhecimentos e vivências, um exemplo do que Gabay e le May definiram em sua pesquisa etnográfica como mindlines, ou guias mentais, que são habitualmente seguidas na prática clínica, em lugar das guidelines formais, baseadas em evidências científicas. Mais do que apenas heurísticas simplificadoras, as mindlines devem ser compreendidas como uma abordagem construtivista do conhecimento, reunindo o saber do grupo de profissionais e as experiências práticas individuais, empregadas de forma tácita e reformuladas constantemente a partir de sua aplicação no cotidiano dos serviços de saúde ${ }^{23}$.

Nos DSC elaborados com base na fala deste grupo de docentes, transparece um conflito quando, a princípio, a HAS é definida como assintomática, mas segue-se a representação da HAS como doença, da qual se esperam sintomas. Evidenciou-se uma contradição entre o conceito formal da HAS como um fator de risco assintomático, assim reafirmado pelas evidências científicas ao longo do tempo, versus a própria experiência prática dos profissionais diante das queixas trazidas pelas pessoas na sua experiência com a doença, e a busca por justificativas palpáveis que demonstrem para as pessoas diagnosticadas a necessidade das intervenções. Nesse sentido, o DSC 3 também sugere a representação da HAS como doença e a relaciona com consequências diretas, em lugar da probabilidade maior de eventos, que podem ou não ocorrer.
Dessa forma, as RS dos docentes de Medicina identificadas nos DSC elaborados trazem consigo um potencial para promoção da doença, abordando um fator de risco como se fora uma doença estabelecida. As possíveis iatrogenias daí resultantes podem acabar percebidas pelos acadêmicos em formação como aceitáveis, apesar dos benefícios incertos do tratamento, especialmente quando não há doença de órgão-alvo ou alto risco cardiovascular definidos.

Como apontado pelos próprios docentes, mas não necessariamente sistematizado no processo de ensino-aprendizagem, sugerimos que, para melhor abordagem da HAS, a estratégia clínica seja exercida com base no Método Clínico Centrado na Pessoa: compreendendo a HAS como um fator de risco e uma variável entre os sinais vitais, explorando a experiência de doença que trouxe a pessoa até o médico e, por fim, buscando com ela a construção de um plano de manejo conjunto $^{10}$. Assim poderemos estabelecer as bases para buscar alternativas eticamente aceitáveis a potenciais iatrogenias produzidas pela medicalização excessiva, conforme a proposta da prevenção quaternária, especialmente no ambiente de ensino.

Os dados analisados no presente estudo retrataram diferentes cenários de prática dos docentes de Medicina, permitindo vislumbrar suas influências sobre os alunos da graduação e sua futura atuação clínica, o que poderia ser aprofundado por uma abordagem etnográfica mais completa, incluindo a observação participante nas atividades docentes. Além da perspectiva das pessoas com HAS, já abordada em diversos estudos, novas pesquisas poderiam também explorar as Representações Sociais em alunos de Medicina e demais cursos de ciências da saúde, assim como dos docentes de outras áreas que lidam com a hipertensão em seu contexto diário, não se limitando à perspectiva médica.

\section{REFERÊNCIAS}

1. Nogueira MI. A reconstrução da formação médica nos novos cenários de prática: inovações no estilo de pensamento biomédico. Physis [online]. 2014. 24( 3 ) [capturado 30 jul 2016]: 909-930. Disponível em: http://www. scielo.br/scielo.php?script=sci_arttext\&pid=S0103-73312014000300909\&lng=en. http://dx.doi.org/10.1590/ S0103-73312014000300013

2. Brasil. Ministério da Saúde. Secretaria de Atenção à Saúde. Departamento de Atenção Básica. Estratégias para o cuidado da pessoa com doença crônica: hipertensão arterial sistêmica. Brasília: Ministério da Saúde, 2013. 128 p. (Cadernos de Atenção Básica, n. 37).

3. James PA et. al. 2014 evidence-based guideline for the management of high blood pressure in adults: report from 
the panel members appointed to the Eighth Joint National Committee (JNC 8). JAMA 2014; 311(5)507-520.

4. Almeida GBS, Paz EPA, Silva GA. Representações sociais sobre hipertensão arterial e o cuidado: o discurso do sujeito coletivo. REME rev. min. enferm [online]. 2013. 17(1) [capturado 15 mai 2015]: 46-53. Disponível em: http:/ /www. reme.org.br/content/imagebank/pdf/en_v17n1a05.pdf

5. Silva MEDC, Moura MEB. Representações sociais de profissionais de saúde sobre a hipertensão arterial: contribuições para a enfermagem. Esc Anna Nery [online]. 2011. 15(1) [capturado 15 mai 2015];75-82. Disponível em: http:// www.scielo.br/scielo.php?script=sci_arttext\&pid=S1414$-81452011000100011 \& \operatorname{lng}=\mathrm{en}$.

6. Silva FM, Budó MLD, Silveira CL, Badke MR, Beuter M. Hipertensão: condição de não doença - o significado da cronicidade na perspectiva dos sujeitos. Texto contexto - enferm. [online]. 2013. 22(1) [capturado 30 jul 2016]; 123-31. Disponível em: http:/ /www.scielo.br/scielo.php?script=sci_ arttext\&pid=S0104-07072013000100015\&lng=en.

7. Fava SMCL, Zago MMF, Nogueira MS, Dázio EMR. Experiência da doença e do tratamento para a pessoa com hipertensão arterial sistêmica: um estudo etnográfico. Rev. Latino-Am. Enfermagem [online]. 2013. 21(5) [capturado 20 set 2017]; [07 telas]. Disponível em:http:// www.scielo.br/scielo.php?script=sci_arttext\&pid=S0104$-11692013000501022 \& \operatorname{lng}=\mathrm{en}$.

8. Lefèvre F; Lefèvre AMC. Depoimentos e Discursos: uma proposta de análise em pesquisa social. Brasília: Liber Livro, 2005.

9. van Den Born BJ, Beutler JJ, Gaillard CA, de Gooijer A, van den Meiracker AH, Kroon AA. Dutch guideline for the management of hypertensive crisis -2010 revision. Neth J Med. [on line]. 2011; 6(5) [capturado 14 mar 2016]; 24855. Disponívelem: https://www.ncbi.nlm.nih.gov/pubmed/21646675

10. Stewart $M$ et al. Medicina centrada na pessoa: transformando o método clínico. 2.ed. Porto Alegre: Artmed; 2010.

11. Marshall IJ; Wolfe CDA; Mckevitt C. Lay perspectives on hypertension and drug adherence: systematic review of qualitative research. BMJ. [on line]. 2012. 345(3953) [capturado 30 jul 2015]; 1-16. Disponívelem: http:/ / www.bmj. com/content/345/bmj.e3953

12. Weiss NS. Relation of High Blood Pressure to Headache, Epistaxis, and Selected Other Symptoms - The United States Health Examination Survey of Adults. N Engl J Med. [online]. 1972. 287(13) [capturado 15 mai 2015]; 631-33. Disponível em: http:/ /www.nejm.org/doi/full/10.1056/ NEJM197209282871303
13. Cantillon P, Morgan M, Dundas R, Simpson J, Bartholomew $\mathrm{J}$, Shaw A et al. Patients perceptions of changes in their blood pressure. J of Hum Hypertension [online]. 1997. 11(4) [capturado 15 mai 2015]; 221-5. Disponível em: https:/ /www. researchgate.net/publication/14033924_Patients\%27_perceptions_of_changes_in_their_blood_pressure

14. Tronvik E, Stovner LJ, Hagen K, Holmen J, Zwart JA. High pulse pressure protects against headache: Prospective and cross-sectional data (HUNT study). Neurology. [on line]. 2008; 70(16) [capturado 15 mai 2015]; 1329-1336. Disponívelem: https://www.ncbi.nlm.nih.gov/m/pubmed/18413586/

15. Hamer M, Batty GD, Stamatakis E, Kivimaki M. Hypertension awareness and psychological distress. Hypertension. [on line]. 2010. 56(3) [capturado 15 mai 2015]; 54750. Disponívelem: http://hyper.ahajournals.org/content/56/3/547.long

16. Gérvas J; Pérez M. São e salvo: e livre de intervenções médicas desnecessárias. Porto Alegre: Artmed, 2016.

17. Jamoulle M, Gomes LF. Prevenção Quaternária e limites em medicina. RevBrasMed Fam Comunidade 2014; 9(31)186-91.

18. Martin AS, Boucher M, Wright JM, Saini V. Mild Hypertension in People at Low Risk. BMJ. [on line]. 2014. 349 [capturado 30 jul 2015]; 1-8. Disponível em: http:/ / www.commed.vcu.edu/Chronic_Disease / hypertension/2015/2Txornot2Tx_BMJ_Sept.pdf

19. Kamran A, SadeghiehAhariS, Biria M, Malepour A, Heydari H. Determinants of Patient's Adherence to Hypertension Medications: Application of Health Belief Model Among Rural Patients. AnMedHeaSci Res. [online]. 2014. 4(6) [capturado 15 mai 2015]; 922-27. Disponível em: https:/ / www.ncbi.nlm.nih.gov/pmc/articles/PMC4250992/

20. Holt E, Joyce C, Dornelles A, Morisky D, Webber LS, Muntner P, Krousel-Wood M. Sex Differences in Barriers to Antihypertensive Medication Adherence: Findings from the Cohort Study of Medication Adherence Among Older Adults (CoSMO). J Am Ger Soc. [on line]. 2013. 61(4) [capturado 15 mai 2015]; 558-64. Disponívelem: http://doi. org/10.1111/jgs.12171

21. Girão ALA, Freitas CHA. Usuários hipertensos na atenção primária à saúde: acesso, vínculo e acolhimento à demanda espontânea. Rev GaúchaEnferm. [on line]. 2016. 37(2) [capturado 20 set, 2017]; e60015. Disponível em: http:// www.scielo.br/scielo.php?script=sci_arttext\&pid=S1983-14472016000200408\&lng=pt. Epub 31-Maio-2016.

22. Corrêa DAS, Felisbino-Mendes MS, Mendes MS, Malta DC, Velasquez-Melendez G. Fatores associados ao uso con- 
traindicado de contraceptivos orais no Brasil. RevSaude Publica [online]. 2017. 51 [capturado 20 set 2017];1-10. Disponível em: www.scielo.br/pdf/rsp/v51/pt_0034-8910-rsp-S1518-87872017051006113.pdf

23. Wieringa S, Greenhalgh T. 10 years of mindlines: a systematic review and commentary. Implementation Science [on line]. 2015. 10:45 [capturado 20 set 2017]; [11 telas]. Disponível em: https://implementationscience.biomedcentral.com/articles/10.1186/s13012-015-0229-x

\section{CONTRIBUIÇÃO DOS AUTORES}

Lucas Zago Scopel e Priscila Soranzo Zappelini contribuíram na elaboração do projeto de pesquisa, coleta e análise de dados, elaboração do texto final.

Carlos Francisco Duarte Júnior contribuiu na elaboração do texto final.

Alessandro da Silva Scholze contribuiu na elaboração do projeto de pesquisa, análise de dados e elaboração do texto final.

\section{CONFLITO DE INTERESSES}

Os autores não identificam nenhum conflito de interesse envolvido na pesquisa.

\section{ENDEREÇO PARA CORRESPONDÊNCIA}

Alessandro da Silva Scholze

Rua Herculano Correa, 84 - ap 202

Centro

Itajaí-SC

CEP 88301-580 